\title{
Tactual localization in cats deprived of cortical areas SI and SII and the dorsal columns'
}

\author{
I. T. Diamond, W. Handall and L. Springer \\ DUKE UNIVERSITY
}

\begin{abstract}
Seven cats were trained in a double grill box to cross when a repeated stimulation of the leg was changed to an alternating stimulation of leg and arm. After learning, cortical areas SI and SII were removed. The cats showed a loss of the habit but could relearn. After reaching the criterion the second time the cats were subjected to a section of the dorsal column and once again all cats relearned the discrimination.
\end{abstract}

Problem

The sensory pathways originating in the spinal cord may be divided into two systems, a refined or epicritic portion, and a primitive or protopathic portion (Head et al., 1905; Rose \& Mountcastle, 1959). The purpose of this inquiry was to remove the epicritic part and test the behavioral capacity of the remaining protopathic projection system. The removal was accomplished in two steps: first, a cortical ablation aimed at producing a total degeneration of VPL, the epicritic thalamic relay nucleus; and second, a complete section of the dorsal columns at a high cervical level. Neither operation alone would be sufficient for our purposes because, on the one hand, the medial lemniscus may have thalamic targets other than VPL (Bowsher, 1961) and the dorsal columns contain fibers to the cerebellum which may provide an important pathway to the cortex via the dentato-rubrothalamic tract. On the other hand, the lateral columns contain an epicritic component which projects in a topographic manner to VPL (Getz, 1952; Perl \& Whitlock, 1961).

As a behavioral indicator of the functional capacity of the remaining protopathic system we selected a learned task in which the cat is required to discriminate between stimuli applied to the arm and leg. This task was chosen because, with the elimination of VPL, the remaining protopathic system should be entirely devoid of a spatially organized representation of the body surface. Such a choice parallels the selection of frequency discrimination as a method for studying the topographic representation of the basilar membrane in the auditory cortex (Butler et al., 1957).

\section{Method}

Seven cats received conditioned avoidance training in a double grill box with the same procedure previously used in studies of the auditory cortex except for the substitution of somatic stimuli. The somatic stimuli consisted of very brief biphasic electrical pulses delivered to subcutaneous electrodes in the arm and leg of the cat. The pulses were continuously presented in groups of three, with 1 second separating the pulses in a group, and 2 seconds separating the groups. Thus the negative stimulus was arm-arm-arm, which was repeated until it was replaced by the positive stimulus, leg-arm-leg. Ten trials were presented each day until the cats learned to avoid shock and then overtraining was continued for an additional month. Before training on the somatic discrimination most cats received conditioned avoidance training on a simpler problem, outset-of-buzzer. After surgery successful performance on the buzzer problem indicated that any further difficulty on the somatic discrimination was not likely the result of nonspecific deficits such as locomotor incapacity.

After the cats learned the somatic discrimination, they were subjected to bilateral removal of the somatic cortex, which was achieved in one stage by subpial aspiration. Following recovery from the cortical surgery the cats were retrained and then subjected to a second operation, a high cervical section of the dorsal columns. Even with every precaution two of the seven cats did not survive the cord surgery. Following recovery from the acute affects of the lesion, the five surviving cats were retrained.

After all training procedures were completed, the cats were killed and perfused with $10 \%$ formalin, the brains and cords were removed, dehydrated in alcohol and embedded in celloidin. The blocks were then sectioned at $50 \mathrm{~m} \mu$. Every 10th section through the cortex was stained with thionin. Additional sections of the thalamus were stained and in some cases every section through VPL was studied. The spinal cords were stained with thionin and with the Holmes stain (Holmes, 1947).

Description of the stimulus. In order to train cats by the same methods used in the auditory studies a technique was developed for presenting stimuli without interfering with the freedom of the cats in crossing back and forth over the partition of a double grill box. This technique involved embedding tantalum wire electrodes beneath the skin of the right arm and leg. The skin was sutured around the circular base of the electrode, leaving a pin permanently exposed above the skin. For each daily session the cats were secured in a harness containing wire leads. One end of the leads was fastened to the exposed pins and the other end was connected through a slip ring with the output of a stimulus generator. As the cat crossed in the grill box the slip ring was pulled back and forth on a track mounted on the ceiling. In this way the cats could be isolated in a sound-treated room while the experimenter, observing from the outside, controlled the presentation of electrical pulses to the arm or leg. 
A cathode follower permitted a continuous monitoring of the voltage between pins. The pulses were viewed on an oscilloscope and the height of the initial phase was taken as the measure of intensity. It appeared that $2 \mathrm{v}$ so measured was above threshold for all cats and yet was clearly not painful. This value therefore was selected as the intensity of the training stimulus.

\section{Results}

Table 1 shows the sessions to a criterion of $17 / 20$ correct on two successive days and indicates the major result: each cat was eventually able to discriminate leg from arm after each operation. The protopathic system alone therefore is sufficient to mediate learned habits based on gross differences in location.

There are some individual differences, however, in the severity of transient effects, and further, there were apparently permanent changes in some cats as a result of the lesions. To some degree these individual differences could be related to the extent of the lesion. For example, after cortical ablation, two cats in which the retrograde degeneration of VPL proved to be most severe and most extensive were unable to relearn at the stimulus intensity used in initial training. In both of these cases, \#50 and \#52, the intensity of the shock to arm and leg had to be increased from $2 \mathrm{v}$ to $2.8 \mathrm{v}$ before relearning occurred. For these cats Table 1 shows the number of trials required to attain criterion at the higher value, $2.8 \mathrm{v}$.

In the three cases, \#50, \#57 and \#47, in which a complete section of the dorsal columns was achieved the resulting locomotor deficit was severe. The symptoms resembled those from which the animals had previously recovered after the first operation only they were more marked. The interruption of projections to the cerebellum likely played a role in the second development of

Table 1. Sessions to Criterion (two consecutive sessions at $85 \%$ or better) on the Auditory and the Somatic Discrimination

\begin{tabular}{rrr} 
Cat & \multicolumn{2}{c}{$\begin{array}{c}\text { Before Surgery } \\
\text { Buzzer }\end{array}$} \\
& $\begin{array}{c}\text { Arm vs } \\
\text { Leg }\end{array}$ \\
50 & 11 & 19 \\
52 & 7 & 10 \\
55 & 9 & 25 \\
17 & - & 38 \\
57 & 8 & 8 \\
199 & - & 9 \\
47 & 9 & 12
\end{tabular}

$$
\begin{array}{cc}
\text { After Ablation of } & \text { After Section of } \\
\text { SI \& SII } & \text { Dorsal Column } \\
\text { Buzzer Arm vs. } & \text { Buzzer Arm vs. }
\end{array}
$$

Leg Leg

* Training discontinued at $80 \%$ performance level because of motor impairments.

** Animal died after achieving $80 \%$ performance level.

- Not tested. the motor syndrome. However, the cats were able to relearn the discrimination even though the arms and legs would slip between the bars of the floor and the cat had to struggle to free himself in order to cross the barrier.

\section{Discussion}

The results provide no evidence for determining whether the reticular formation, the tectum, the ventral thalamus, the intralaminar thalamic nuclei, or some combination of target areas of the lateral columns is responsible for the residual capacity (Nauta \& Kuypers, 1958). However, it is worth noting that in three of the cats reported here, \#50, \#57 and \#47, cortical potentials in the anterior ectosylvian gyrus could be evoked by somatic stimulation (Erickson \& Diamond, unpublished experiments). The posterior nuclei of the dorsal thalamus apparently relay impulses from the lateral columns to the vicinity of auditory area II (Berman, 1961).

\section{References}

BERMAN, A. L. Overlap of somatic and auditory cortical response fields in anterior ectosylvian gyrus of cat. J. Neurophysiol., 1961, 24, 595-607.

BOWSHER, D. The termination of secondary somatosensory neurons within the thalamus of Macaca mulatta: An experimental degeneration study. J. comp. Neurol., 1961, 117, 213-227.

BUTLER, R. A., DIAMOND, I. T., \& NEFF, W. D. Role of auditory cortex in discrimination of changes of frequency. J. Neurophysiol., 1957, 20, 108-120.

GETZ, B. The termination of spinothalamic fibres in the cat as studied by the method of terminal degeneration. Acta Anat., 1952, 16, 271-290.

HEAD, H., RIVERS, W., \& SHERREN, J. The afferent nervous system from a new aspect. Brain, 1905, 28, 98-115.

HOLMES, W. In Recent Advances in Clinical Pathology. Philadelphia: Blakiston Co., 1947.

NAUTA, W. J. H. , \& KUYPERS, G. J. M. Some ascending pathways in the brain stem reticular formation. In Jasper, H. H. et al. (Eds.), Reticular formation of the brain. Boston: Little, Brown, 1958. Pp.1-30.

PERL, E. R., \& WHITLOCK, D. G. Somatic stimuli exciting spino-thalamic projections to thalamic neurons in cat and monkey. Exp. Neurol., 1961, 3, 256-296.

ROSE, J. E., \& MOUNTCASTLE, V. B. Touch and kinesthesis. In Field, J. etal. (Eds.), Handbook of physi ology, Vol. I. Washington, D. C.: American Physiological Society, 1959. Pp. 387-429.

\section{Note}

1. This research was supported by grants from the National Science Foundation (G-7020) and the National Institute of Mental Health (M-4849). 